\title{
Frequencies of CP2 stars in open clusters and the galactic field
}

\author{
M. Rode-Paunzen, H. Pöhnl, E. Paunzen and H. M. Maitzen \\ Institute for Astronomy, University of Vienna, Türkenschanzstr. 17, A-1180 Vienna, Austria. \\ email: rode@astro.univie.ac.at
}

\begin{abstract}
The Michigan Catalogue (Vol. I - V) is an excellent database to study the distribution of CP2 stars in the galactic field in the Southern hemisphere. A cross-check with the BidelmanCatalogue (1973) and the "Catalogue Général des Étoiles Ap et Am" (Renson 1991) shows that in the Michigan Catalogue $3.6 \%$ of all stars in the relevant spectral region are CP2 stars. Any discrepant classified stars could be verified photometrically in the $\Delta a$-system. The distribution of CP2 stars does not show any particular preferred region in the Southern hemisphere.

The ages of these stars and an evolutionary dependence of the CP2 effect was another important question. We conclude that the CP2 stars occupy the full Main Sequence band.
\end{abstract}

Keywords. Stars: chemically peculiar, evolution, catalogs, methods: statistical

\section{Frequency of CP2 stars in the galactic plane}

\subsection{Introduction}

Magnetic chemically peculiar stars, the so-called magnetic Ap or CP2 stars, are on the upper Main Sequence. The CP2 stars shows overabundances of one or more chemical elements like silicon, strontium, chromium and the Rare Earth europium in their spectra. CP2 stars show one or more flux depressions centered near $\lambda 4100, \lambda 5200$, and $\lambda 6300$. These flux depressions are most certainly a consequence of the nonsolar elemental abundance of CP2 stars and can be used to identify such objects via $\Delta a$ photometry. This narrow-band, three filter photometric system measures the flux depression at $\lambda 5200$ by sampling the depth of this flux depression. It compares the flux at the center with the adjacent regions at $\lambda 5000$ and $\lambda 5500$ using a band-width of $130 \AA$.

\subsection{Catalogues}

The University of Michigan Catalogue of Two-Dimensional Spectral Types for the HD Stars as published by Houk \& Cowley (1975), Houk (1978, 1982), Houk \& Smith-Moore (1988), and Houk \& Swift (1999) includes more than 50000 late B, A and early F stars of the Southern hemisphere, brighter than $V=12$ mag. A search for bona fide or possible magnetic chemically peculiar stars in this catalogue results in a percentage of $3.6 \%$ of objects which could be classified as more or less peculiar. Within this "peculiar subsample", we find $44 \%$ B-type, 52\% A-type and $4 \%$ early F-type objects. A cross-check of the photometric Geneva catalogue of Rufener (1988) and the Catalogue of CP and Am stars of Renson (1991) with the published $\Delta a$ values gives an comprehensive database for statistical studies. 

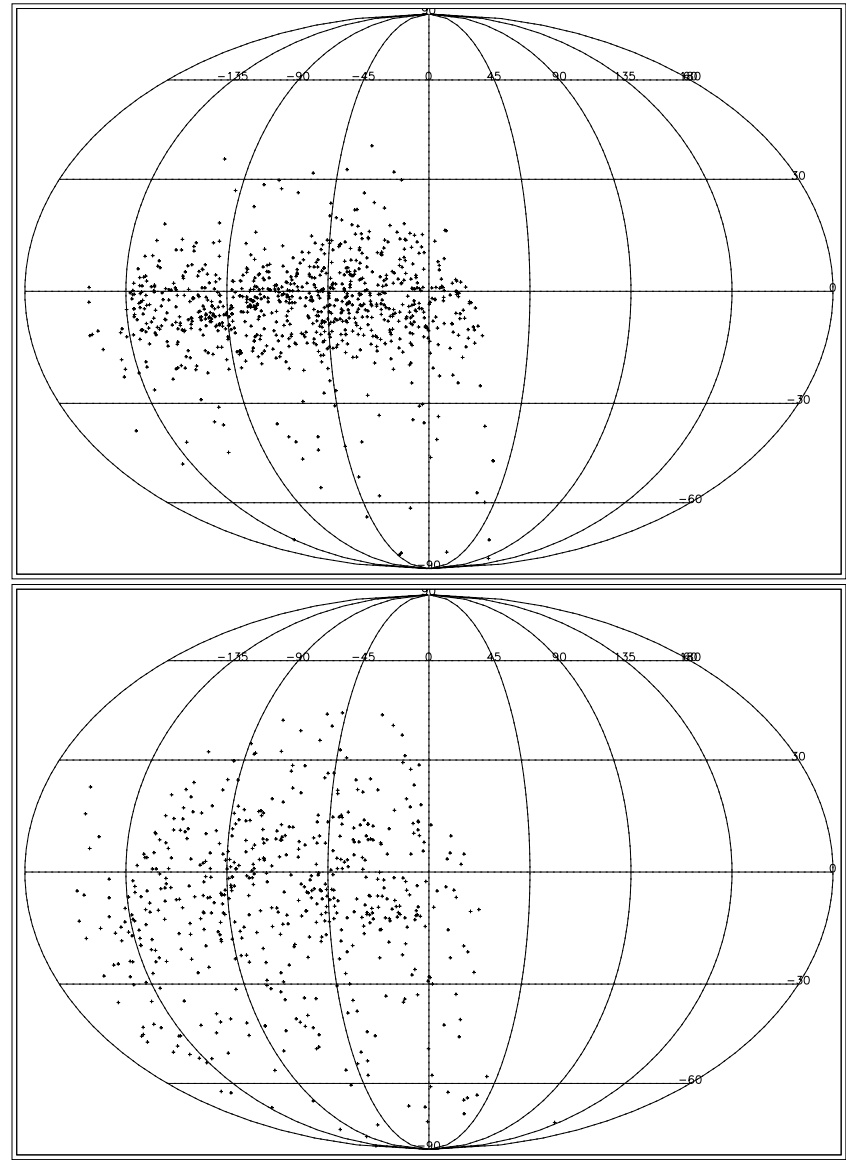

Figure 1. Distribution of the galactic coordinates for the $\mathrm{Si}$ and $\mathrm{Si}+$ group (upper panel) as well as the $\mathrm{Sr}$ and $\mathrm{SrCrEu}$ group (lower panel).
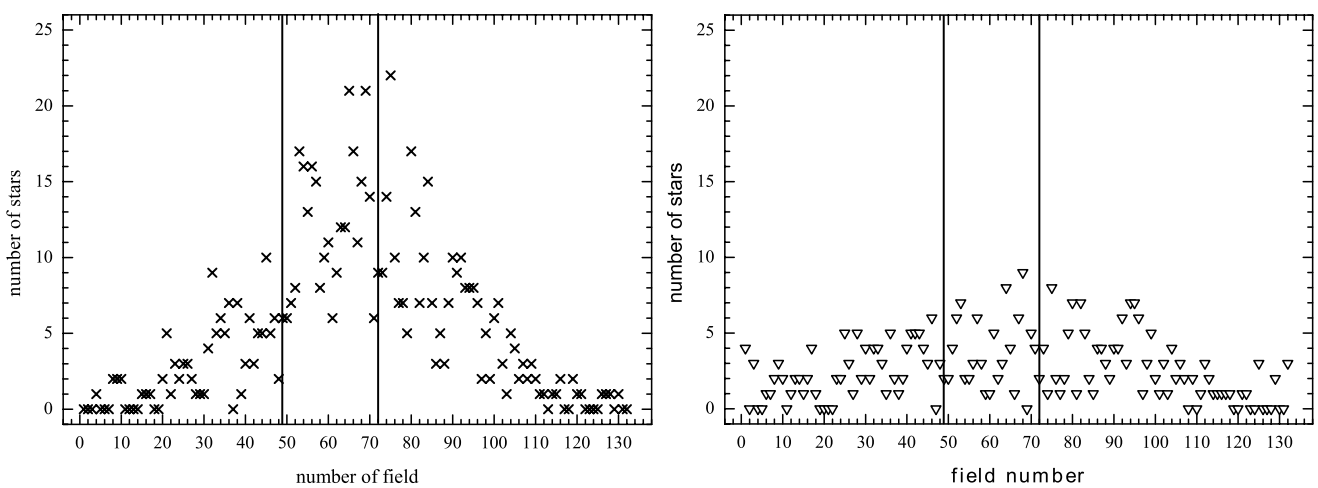

Figure 2. Partition numbers 49 to 72 are in the region of the galactic plane $\left(-5^{\circ}<b<5^{\circ}\right)$ and are marked with straight lines for the $\mathrm{Si}$ and $\mathrm{Si}+$ group (left panel) as well as the $\mathrm{Sr}$ and $\mathrm{SrCrEu}$ group (right panel). 
Table 1. Objects with discrepant classifications.

\begin{tabular}{|c|c|c|c|c|c|c|c|c|}
\hline $\mathrm{HD}$ & DM & $\alpha_{1900}$ & $\delta_{1900}$ & $\mathrm{~m}_{\text {phot }}$ & Michigan & Bidelman & Renson & $\mathrm{m}_{V}$ \\
\hline - & & & & & & & & - \\
\hline 19712 & BD-02 563 & $\begin{array}{lll}305 & 13,9\end{array}$ & $\begin{array}{lll}-02 & 04 & 29\end{array}$ & 7,32 & B9 $\mathrm{V}$ & $\mathrm{CrEu}$ & $\mathrm{A} 0 \mathrm{Cr} \mathrm{Eu}$ & \\
\hline 21201 & CP-52 394 & 3208,6 & $\begin{array}{lll}-52 & 14 & 53\end{array}$ & 9,1 & A1MA8-A9 & $\mathrm{SrCr}$ & A $1-A 9$ & 8,9 \\
\hline 24212 & CP-79 114 & 346,1 & -7953 & 9,3 & B9 III/IV & $\mathrm{SiCr}$ & $\mathrm{A} 0 \mathrm{Si} \mathrm{Cr}$ & 9,6 \\
\hline 32145 & $\mathrm{BD}+03739$ & $455 \quad 50,4$ & +033410 & 7,11 & B8 II & $\mathrm{Si}$ & $\mathrm{B} 8 \mathrm{Si}$ & \\
\hline 32966 & BD-14 1045 & 50148,8 & -144951 & $6,97 \mathrm{~V}$ & B8 II & $\mathrm{Si}$ & B9 $\mathrm{Si}$ & 7,1 \\
\hline 33917 & BD-02 1176 & $508 \quad 25,5$ & -024443 & 9,8 & $\mathrm{~A} 0 \mathrm{~V}$ & $\mathrm{Si}$ & $\mathrm{AO} \mathrm{Si}$ & \\
\hline 34607 & BD-12 1126 & $\begin{array}{lll}5 & 13 & 35,9\end{array}$ & -123554 & 10,4 & AO V & $\mathrm{Si}$ & $\mathrm{AO} \mathrm{Si}$ & 9,8 \\
\hline 35177 & $\mathrm{BD}+01991$ & 51750,8 & +013604 & 8,4 & $\mathrm{~B} 8 / 9 \mathrm{~V}$ & $\mathrm{Si}$ & B9 Si & \\
\hline 36668 & $\mathrm{BD}+001113$ & $\begin{array}{lll}5 & 28 & 17,3\end{array}$ & +003302 & 8,06 & B8 II A & $\mathrm{Si}$ & B7 HE FBL & \\
\hline 36997 & BD-02 1305 & $\begin{array}{lll}5 & 30 & 12\end{array}$ & $-02 \quad 2650$ & 8,31 & B9 II & $\mathrm{Si}$ & B9 $\mathrm{Si} \mathrm{Sr}$ & \\
\hline 40146 & BD-03 1243 & 55200,3 & -034608 & 9,7 & B9 III/IV & $\mathrm{Si}$ & $\mathrm{AO} \mathrm{Si}$ & \\
\hline 40966 & BD-07 1255 & $\begin{array}{lll}5 & 57 & 05,3\end{array}$ & -075804 & 10,4 & B8 II & $\mathrm{Si}$ & B9 Si & \\
\hline 43408 & $\mathrm{BD}+001352$ & $\begin{array}{lll}6 & 10 & 55,7\end{array}$ & +005431 & 8,1 & B9 $\mathrm{V} \mathrm{A}$ & SrEu & B9 $\mathrm{Sr} \mathrm{Eu}$ & \\
\hline 45931 & BD-00 1312 & $\begin{array}{ll}62520,9\end{array}$ & +001818 & 8,8 & B9 V & $\mathrm{Si}$ & $\mathrm{AO} \mathrm{Si}$ & \\
\hline 47026 & $\mathrm{CO}-482371$ & $\begin{array}{lll}631 & 18,9\end{array}$ & $\begin{array}{llll}-48 & 04 & 18\end{array}$ & 9,2 & B9 V & $\mathrm{SrCrEu}$ & B9 $\mathrm{Sr} \mathrm{Cr} \mathrm{Eu}$ & 9,6 \\
\hline 47634 & BD-00 1380 & 63431,1 & +004359 & 8,8 & $\mathrm{~B} 9 \mathrm{IV} / \mathrm{V}$ & $\mathrm{Si}$ & $\mathrm{A} 0 \mathrm{Si}$ & \\
\hline 48160 & BD-08 1521 & 63652 & $\begin{array}{lll}-08 & 25 & 45\end{array}$ & 9,7 & B9 III/IV & $\mathrm{Si}$ & $\mathrm{AO} \mathrm{Si}$ & \\
\hline 51088 & $\mathrm{CO}-244586$ & $\begin{array}{lll}650 & 46,3\end{array}$ & $\begin{array}{llll}-24 & 35 & 26\end{array}$ & 7,9 & B9 V & $\mathrm{Si}$ & B8 Si ? & 8,3 \\
\hline 52669 & BD-14 1687 & $\begin{array}{lll}6 & 57 & 1,7\end{array}$ & -145432 & 8,76 & B9 II/III & $\mathrm{Si}$ & $\mathrm{A} 0 \mathrm{Si}$ & 9,1 \\
\hline 53204 & $\mathrm{BD}+011663$ & $\begin{array}{lll}659 & 05,3\end{array}$ & 013918 & 8,2 & B9 III A & $\mathrm{Si}$ & B9 $\mathrm{Si}$ & \\
\hline 54288 & $\mathrm{CO}-452928$ & $703 \quad 7,1$ & $\begin{array}{lll}-45 & 0636\end{array}$ & 9,7 & $\mathrm{G} 8 / \mathrm{K} 1(\mathrm{II})+\mathrm{A} / \mathrm{F}$ & Sr GBand & F2 Sr & 10,0 \\
\hline 54772 & $\mathrm{CO}-333532$ & $\begin{array}{lll}7 & 05 & 03,2\end{array}$ & -335359 & 8,2 & $\mathrm{AO} \mathrm{V}$ & $\mathrm{Si}$ & $\mathrm{AO} \mathrm{Si}$ & 8,9 \\
\hline 55839 & $\mathrm{CO}-353417$ & $\begin{array}{lll}7 & 09 & 29,9\end{array}$ & $\begin{array}{lll}-35 & 28 & 54\end{array}$ & 9,7 & A3 & $\mathrm{Sr}$ & A $3 \mathrm{Sr}$ & 10,2 \\
\hline 58443 & $\mathrm{CO}-363586$ & 72029,1 & $\begin{array}{lll}-36 & 28 & 24\end{array}$ & 8,9 & $\mathrm{~A} 4 \mathrm{~V}$ & $\mathrm{SrCrEu}$ & A3 $\mathrm{Sr} \mathrm{Cr} \mathrm{Eu}$ & 9,1 \\
\hline 59758 & $\mathrm{CO}-373624$ & $\begin{array}{lll}7 & 26 & 17,9\end{array}$ & -375154 & 10,5 & A0 II & $\mathrm{Si}$ & $\mathrm{A} 0 \mathrm{Si}$ & 10,9 \\
\hline 60210 & $\mathrm{BD}+001991$ & $\begin{array}{lll}728 & 21,3\end{array}$ & +002905 & 8,9 & B9 V & SrEu & A2 $\mathrm{Sr} \mathrm{Eu}$ & \\
\hline 61260 & BD-21 2042 & $\begin{array}{lll}733 & 17,9\end{array}$ & $\begin{array}{lll}-21 & 30 & 53\end{array}$ & 9,8 & B8/9 II & $\mathrm{Si}$ & B9 Si ? & 10,1 \\
\hline 61382 & BD-04 2031 & 73355,9 & -043400 & 8,5 & B9 V & $\mathrm{Si}$ & B9 $\mathrm{Si}$ & \\
\hline 62005 & BD-22 1980 & 73648,5 & $\begin{array}{lll}-22 & 28 & 10\end{array}$ & 9,8 & B9 II & $\mathrm{Si}$ & $\mathrm{A} 1 \mathrm{Si}$ & 10,2 \\
\hline 62512 & BD-00 1799 & 73909,2 & +002852 & 8,8 & $\mathrm{~A} 0 \mathrm{~V}$ & SrEu & $\mathrm{AO} \mathrm{Sr} \mathrm{Eu}$ & \\
\hline 63843 & BD-05 2269 & $745 \quad 52,9$ & -054747 & 9,7 & A1 (IV) & $\mathrm{CrEu}$ & $\mathrm{AO} \mathrm{Cr} \mathrm{Eu}$ & \\
\hline 64901 & $\mathrm{CO}-403618$ & 75056 & $\begin{array}{llll}-40 & 35 & 41\end{array}$ & 8,4 & $\mathrm{~B} 8 / 9 \mathrm{II} / \mathrm{III}$ & $\mathrm{Si}$ & $\mathrm{AO} \mathrm{Si}$ & 8,6 \\
\hline 64972 & $\mathrm{CO}-274729$ & $\begin{array}{lll}7517,5 & 17\end{array}$ & $\begin{array}{llll}-28 & 01 & 10\end{array}$ & 7,3 & $\mathrm{~B} 8 / 9$ II & $\mathrm{Si}$ & B8 Si & 7,2 \\
\hline 64988 & BD-09 2289 & $\begin{array}{lll}751 & 21,8\end{array}$ & $\begin{array}{lll}-09 & 35 & 52\end{array}$ & 7,8 & B9 IV/V & $\mathrm{Si}$ & $\mathrm{AO} \mathrm{Si}$ & \\
\hline 65941 & BD-16 2242 & $\begin{array}{lll}7 & 56 & 07,1\end{array}$ & $\begin{array}{llll}-16 & 17 & 36\end{array}$ & 8,9 & B9 IV & $\mathrm{Si}$ & B9 Si ? & 9,8 \\
\hline 65943 & $\mathrm{CO}-265342$ & $\begin{array}{lll}756 & 10,5\end{array}$ & $\begin{array}{lll}-26 & 13 & 36\end{array}$ & 9,2 & A $0 \mathrm{~V}$ & SrEu & A2 $\mathrm{Sr} \mathrm{Eu}$ & 9,6 \\
\hline 66273 & BD-20 2345 & 75734,1 & $-2034 \quad 52$ & 8,7 & B9 IV & $\mathrm{Si}$ & $\mathrm{B} 9 \mathrm{Si} ?$ & 8,8 \\
\hline 67982 & $\mathrm{CO}-444089$ & $805 \quad 17,2$ & -445030 & 9,3 & A1 IV & $\mathrm{Si}$ & B9 Si & 10,0 \\
\hline 68326 & $\mathrm{CO}-493382$ & 80642 & -495543 & 8,9 & $\mathrm{~B} 9 \mathrm{IV} / \mathrm{V}$ & $\mathrm{Si}$ & $\mathrm{A} 0 \mathrm{Si}$ & 9,7 \\
\hline 68826 & $\mathrm{CO}-483586$ & $808 \quad 54,1$ & $\begin{array}{lll}-48 & 26 & 47\end{array}$ & $9,2 \mathrm{~V}$ & B9 III & $\mathrm{Si}$ & B9 Si & 9,3 \\
\hline 69193 & $\mathrm{CO}-473716$ & 81040,6 & $\begin{array}{lll}-47 & 28 & 10\end{array}$ & 9,6 & B9 II & $\mathrm{Si}$ & $\mathrm{AO} \mathrm{Si}$ & 10,3 \\
\hline 69932 & $\mathrm{CO}-453963$ & $8 \quad 14 \quad 09,9$ & $\begin{array}{lll}-45 & 22 & 42\end{array}$ & 9,6 & B9 III & $\mathrm{Si}$ & B9 Si & 10,1 \\
\hline 70325 & $\mathrm{CO}-295976$ & $\begin{array}{lll}8 & 16 & 13,3\end{array}$ & $\begin{array}{lll}-29 & 13 & 28\end{array}$ & 7,3 & B9 IV & $\mathrm{Si}$ & $\mathrm{AO} \mathrm{Si}$ & 7,3 \\
\hline 72770 & $\mathrm{CO}-27 \quad 5635$ & 82932,6 & $-27 \quad 4732$ & 8,8 & A $2 \mathrm{IV} / \mathrm{V}$ & $\mathrm{CrEu}$ & $\mathrm{A} 0 \mathrm{Cr} \mathrm{Eu}$ & 9,1 \\
\hline 74041 & $\mathrm{CO}-414363$ & 83628,3 & $\begin{array}{lll}-41 & 34 & 48\end{array}$ & 9,7 & AO V & $\mathrm{Si}$ & A0 Si & 10 \\
\hline 78201 & CO-34 5573 & 90148,5 & $\begin{array}{lll}-34 & 08 & 03\end{array}$ & 8,3 & $\mathrm{~B} 8 / 9 \mathrm{IV}$ & SrEu & AO Sr Eu & 8,1 \\
\hline 79718 & CP-56 2052 & 910,5 & -5615 & 9,7 & $\mathrm{~A} 0 / 1 \mathrm{VN}$ & $\mathrm{Si}$ & A Si & 9,7 \\
\hline 84629 & CP-55 2483 & 941,4 & -5513 & 9,5 & B9,5 II & $\mathrm{Si}$ & B9 Si & 9,4 \\
\hline 84907 & CP-54 2700 & 943,3 & -5453 & 8,9 & $\mathrm{~A} 0 \mathrm{IV} / \mathrm{V}$ & $\mathrm{Si}$ & $\mathrm{AO} \mathrm{Si}$ & 8,9 \\
\hline 87432 & $\mathrm{CO}-455717$ & 95944,7 & $\begin{array}{llll}-45 & 16 & 44\end{array}$ & 8,9 & A2 IV & $\mathrm{CrEu}$ & $\mathrm{AO} \mathrm{Cr} \mathrm{Eu}$ & 9,1 \\
\hline 88242 & $\mathrm{CO}-514511$ & $1005 \quad 18,5$ & $\begin{array}{llll}-51 & 37 & 50\end{array}$ & 8,9 & $\mathrm{~B} 9,5 \mathrm{~V}$ & $(*)$ & $\mathrm{AO} \mathrm{Si} \mathrm{Cr}$ & 9,5 \\
\hline 91756 & $\mathrm{CO}-38 \quad 6572$ & 103036,5 & $\begin{array}{lll}-39 & 02 & 09\end{array}$ & 8,4 & FM DELTA DEL & SrEu & A0 Sr Eu & 8,7 \\
\hline 92385 & CP-64 1374 & 1034,8 & -6432 & 6,6 & $\mathrm{~B} 8 / 9 \mathrm{~V}$ & $\mathrm{Si}$ & $\mathrm{B} 9 \mathrm{Si}$ & 6,7 \\
\hline 95442 & $\mathrm{CO}-258385$ & $1055 \quad 53,5$ & $-25 \quad 1048$ & 8,2 & $\mathrm{~A} 1 \mathrm{~V}(\mathrm{M})$ & $\mathrm{SrCr}$ & $\mathrm{AO} \mathrm{Sr} \mathrm{Cr} \mathrm{Eu}$ & 7,9 \\
\hline 101724 & CP-63 1944 & 1137,2 & -6314 & 7,5 & B9 II/III & $\mathrm{Si}$ & B9 Si & 8,0 \\
\hline 103671 & CP-55 4727 & 1151,1 & -5553 & 8,7 & B8 III & $\mathrm{Si}$ & $\mathrm{B} 8 \mathrm{Si}$ & 8,7 \\
\hline 105379 & $\mathrm{CO}-309691$ & 120252,1 & $\begin{array}{lll}-30 & 35 & 2\end{array}$ & 8,1 & A0 $\mathrm{V}$ & $\mathrm{SrCr}$ & $\mathrm{AO} \mathrm{Sr} \mathrm{Cr}$ & 8 \\
\hline 115000 & CP-61 3532 & 139,1 & -6151 & 9,8 & B9 III & $\mathrm{Si}$ & B9 Si & 9,4 \\
\hline 117055 & $\mathrm{CO}-478353$ & 132236,1 & -475325 & 9 & B9 III & $\mathrm{Si}$ & B8 Si & 9,4 \\
\hline 120059 & CP-58 5187 & 1342 & -5818 & 08,8 & B9 III & $\mathrm{Si}$ & B8 Si & 8,8 \\
\hline 121208 & CP-57 6394 & 1348,9 & -5757 & 9,5 & A9 V & $\mathrm{Sr}$ & B9 Sr & 9,4 \\
\hline 126198 & CP-52 7213 & 1418,9 & -5300 & 8 & B $8 / 9$ II & $\mathrm{Si}$ & $\mathrm{B} 9 \mathrm{Si}$ & 8,0 \\
\hline 128075 & CP-66 2588 & 1429,7 & -6654 & 8,8 & B9 II/III & $\mathrm{Si}$ & B9 $\mathrm{Si}$ & 8,4 \\
\hline 128997 & CP-66 2616 & 1435 & -6621 & 8,9 & B9 V & $\mathrm{Si}$ & B9 Si & 8,9 \\
\hline 129460 & CP-53 6083 & 1437,5 & -5406 & 10,1 & B9 III & $\mathrm{Si}$ & B9 Si & 9,9 \\
\hline 132319 & CP-53 6186 & 1453,3 & -5340 & 9,6 & $\mathrm{~A} 0 / 1 \mathrm{~V}$ & $\mathrm{Si}$ & A0 $\mathrm{Si} \mathrm{Sr}$ & 9,3 \\
\hline 133428 & CP-76 977 & 1459,2 & -7634 & 9 & A2 $\mathrm{V}$ & $\mathrm{Si}$ & $\mathrm{AO} \mathrm{Si}$ & 9,1 \\
\hline 133755 & CP-52 7861 & 1501,1 & $\begin{array}{llll}-52 & 29 & 30\end{array}$ & 8,7 & B9 II/III & $\mathrm{Si}$ & B9 Si & 8,7 \\
\hline 134185 & $\mathrm{CO}-518801$ & $\begin{array}{lll}15 & 03 & 14,1\end{array}$ & $\begin{array}{lll}-51 & 46 \quad 57\end{array}$ & 9,6 & $\mathrm{Fo} / 2 \mathrm{~V}$ & $\mathrm{Si}$ & FO Si & 9,1 \\
\hline 138218 & BD-05 4089 & $\begin{array}{lll}15 & 25 & 31,9\end{array}$ & $\begin{array}{lll}-05 & 27 & 47\end{array}$ & 9,3 & $\mathrm{~A} 3 / 5$ II & $\mathrm{Sr}$ & $\mathrm{A} 2 \mathrm{Sr}$ & \\
\hline 138777 & BD-06 4237 & $\begin{array}{lll}15 & 29 & 07,8\end{array}$ & $\begin{array}{lll}-06 & 33 & 08\end{array}$ & 9,4 & F2 IV & SrEu & A3 $\mathrm{Sr} \mathrm{Eu}$ & \\
\hline 141100 & CP-65 3150 & 1542,1 & -6531 & 9,4 & A 1 MA6-A9 & $\mathrm{Cr}$ & A $1-A 9$ & 8,9 \\
\hline 142070 & BD-00 3026 & $\begin{array}{lll}15 & 47 & 25,9\end{array}$ & +004355 & 8,3 & B9 $\mathrm{V}$ & $\mathrm{SrCrEu}$ & $\mathrm{AO} \mathrm{Sr} \mathrm{Cr}$ & \\
\hline 142778 & CP-60 6256 & 1551,2 & -6023 & 9,3 & B9 II & $\mathrm{Si}$ & $\mathrm{B} 8 \mathrm{Si}$ & 10,0 \\
\hline 142884 & $\mathrm{CO}-2312597$ & 155152 & $\begin{array}{llll}-23 & 14 & 14\end{array}$ & $6,8 \mathrm{~V}$ & B8/9 III & $\mathrm{Si}$ & B9 $\mathrm{Si}$ & 6,8 \\
\hline 142960 & CP-60 6280 & 1552,4 & -6042 & 9,3 & B9 IV & $\mathrm{SrCrEu}$ & $\mathrm{A} 0 \mathrm{Sr} \mathrm{Cr} \mathrm{Eu}$ & 9,7 \\
\hline 143881 & $\mathrm{CO}-4410603$ & 155743,2 & $\begin{array}{lll}-45 & 03 & 47\end{array}$ & 10,4 & B8 II/III & $\mathrm{Si}$ & B9 $\mathrm{Si}$ & 10,9 \\
\hline 146971 & BD-09 4353 & $\begin{array}{lll}16 & 14 & 02,1\end{array}$ & -092257 & 8,7 & AO III/IV (p) & SrCrEu & $\mathrm{AO} \mathrm{Sr} \mathrm{Cr}$ & \\
\hline 146998 & $\mathrm{CO}-2511477$ & $16 \quad 14 \quad 4,4$ & -253654 & $9,5 \mathrm{~V}$ & $\mathrm{~A} 8 / 9 \mathrm{~V}$ & $\mathrm{Sr}$ & $\mathrm{A} 6 \mathrm{Sr} \mathrm{Cr}$ & 9,5 \\
\hline 149046 & BD-06 4450 & $\begin{array}{lll}16 & 27 & 04,3\end{array}$ & $\begin{array}{lll}-06 & 58 & 15\end{array}$ & 9,6 & $\mathrm{~B} 9,5 \mathrm{~V}$ & $\mathrm{SrCrEu}$ & $\mathrm{A} 0 \mathrm{Sr} \mathrm{Cr}$ & \\
\hline 149334 & $\mathrm{CO}-3311273$ & $\begin{array}{lll}16 & 28 & 52,7\end{array}$ & $\begin{array}{lll}-33 & 48 & 17\end{array}$ & 9,2 & A9 IV & $\mathrm{Sr}$ & $\mathrm{A} 6 \mathrm{Sr}$ & 9,1 \\
\hline 149923 & CP-60 6605 & 1632,6 & -6023 & 8,7 & AO II & $\mathrm{CrEu}$ & B9 $\mathrm{Cr} \mathrm{Eu}$ & 8,9 \\
\hline
\end{tabular}

The Michigan-catalogue describes the characteristic spectral lines which are typical for most of CP stars. Therefore we have divided the sample in the four groups defined in 
Table 2. Table 1 - contiued

\begin{tabular}{|c|c|c|c|c|c|c|c|c|}
\hline $\mathrm{HD}$ & DM & $\alpha_{1900}$ & $\delta_{1900}$ & $\mathrm{~m}_{\text {phot }}$ & Michigan & Bidelman & Renson & $\mathrm{m}_{V}$ \\
\hline 150592 & $\mathrm{CO}-4610973$ & 163656 & $-4652 \quad 29$ & 8,5 & $\mathrm{~A} 0 / 1 \mathrm{~V}$ & $\mathrm{Si}$ & $\mathrm{B} 9 \mathrm{Si}$ & 8,7 \\
\hline 152044 & CP-52 10302 & $\begin{array}{lll}16 & 45 & 49,7\end{array}$ & $\begin{array}{lll}-52 & 35 & 59\end{array}$ & 8,7 & $\mathrm{~A} 1 \mathrm{~V}$ & SrCrEu & A0 $\mathrm{Sr} \mathrm{Cr} \mathrm{Eu}$ & 9,0 \\
\hline 156791 & CP-52 10606 & $17 \quad 1425,5$ & $\begin{array}{lll}-52 & 25 & 17\end{array}$ & 9,2 & B9 III/IV & $\mathrm{Si}$ & $\mathrm{A} 0 \mathrm{Si}$ & 9,9 \\
\hline 159545 & BD-02 4402 & $1730 \quad 17,3$ & -024933 & 7,5 & B8 II & $\mathrm{Si}$ & B9 Si & \\
\hline 162306 & CO-35 11994 & $17 \quad 45 \quad 14$ & $\begin{array}{lll}-35 & 03 & 18\end{array}$ & 8,3 & B8 IV & $\mathrm{Si}$ & B9 Si & 8,9 \\
\hline 162651 & $\mathrm{BD}+013525$ & 174655,9 & +010728 & 7,9 & B9 IV & $\mathrm{Si}$ & $\mathrm{AO} \mathrm{Si}$ & \\
\hline 164827 & BD-00 3404 & 175752,8 & +002724 & 9,4 & B9 V & $\mathrm{CrEu}$ & $\mathrm{AO} \mathrm{Cr} \mathrm{Eu}$ & \\
\hline 166053 & BD-19 4871 & $\begin{array}{lll}18 & 03 & 42,2\end{array}$ & $\begin{array}{lll}-19 & 22 & 23\end{array}$ & 8,2 & B9 IB/II & $\mathrm{Si}$ & B9 Si ? & 8,3 \\
\hline 166596 & CO-41 12534 & 18067,7 & -412133 & 5,3 & B2 III & $\mathrm{Si}$ & $\mathrm{B} 3 \mathrm{Si}$ & 5,5 \\
\hline 166968 & $\mathrm{CO}-27 \quad 12588$ & $1807 \quad 53,8$ & -273145 & 7,4 & B8 II/III & $\mathrm{Si}$ & B9 $\mathrm{Si}$ & 7,2 \\
\hline 167444 & $\mathrm{CO}-42 \quad 13073$ & 18105,1 & $-42 \quad 1456$ & 8,8 & B9 IV & $\mathrm{Si}$ & $\mathrm{A} 0 \mathrm{Si}$ & 9,2 \\
\hline 168163 & BD-16 4806 & $1813 \quad 17,5$ & -162026 & 8,9 & B8 III & $\mathrm{Si}$ & $\mathrm{B} 4 \mathrm{Si} ?$ & 8,7 \\
\hline 171279 & BD-07 4623 & 182847 & $\begin{array}{llll}-07 & 47 & 19\end{array}$ & 7,3 & B9 V A & SrEu & $\mathrm{Ao} \mathrm{Sr} \mathrm{Cr}$ & \\
\hline 172032 & BD-16 4963 & 183250,5 & $\begin{array}{lll}-16 & 23 & 45\end{array}$ & 8,2 & FM DELTA DEL & $\mathrm{Sr}$ & A9 $\mathrm{Sr} \mathrm{Cr}$ & 7,7 \\
\hline 173612 & BD-08 4699 & 184104 & $\begin{array}{lll}-08 & 32 & 19\end{array}$ & 9,5 & $\mathrm{~B} 9 \mathrm{IV} / \mathrm{V}$ & $\mathrm{Si}$ & $\mathrm{AO} \mathrm{Si}$ & \\
\hline 174595 & BD-20 5297 & 18467,6 & -204309 & 8,8 & B9 II & $\mathrm{Si}$ & $\mathrm{AO} \mathrm{Si}$ & 9,1 \\
\hline 174646 & BD-013581 & 184628 & $\begin{array}{llll}-01 & 09 & 45\end{array}$ & 8,1 & B8/9 II & $\mathrm{Si}$ & B9 Si & \\
\hline 176332 & BD-20 5358 & 185430,7 & $\begin{array}{llll}-20 & 01 & 16\end{array}$ & 8,9 & B9 III & $\mathrm{Si}$ & $\mathrm{B} 8 \mathrm{Si}$ & 9,3 \\
\hline 176555 & $\mathrm{CO}-4512940$ & $1855 \quad 43,9$ & $\begin{array}{lll}-45 & 51 & 31\end{array}$ & 7,1 & B9 (III) & $\mathrm{Si}$ & B9 Si & 8,0 \\
\hline 179902 & BD-21 5306 & 190912 & -215504 & 10,1 & AO III & $\mathrm{SrCrEu}$ & $\mathrm{A} 1 \mathrm{Sr} \mathrm{Cr} \mathrm{Eu}$ & 10 \\
\hline 180058 & BD-11 4921 & 190952 & -115318 & 9,5 & $\mathrm{~A} 7 / 8 \mathrm{~V}$ & $\mathrm{Sr}$ & A3 $\mathrm{Sr}$ & \\
\hline 182340 & BD-05 4959 & $\begin{array}{lll}19 & 19 & 03,6\end{array}$ & -053726 & 9,4 & B8/9 II & $\mathrm{Si}$ & $\mathrm{AO} \mathrm{Si}$ & \\
\hline 185129 & BD-16 5383 & 193229,5 & $\begin{array}{llll}-16 & 01 & 53\end{array}$ & 9,2 & B9 IV/V & $\mathrm{CrEu}$ & B9 Cr Eu & 9,9 \\
\hline 185280 & $\mathrm{CO}-41 \quad 13623$ & $1933 \quad 17,8$ & $\begin{array}{lll}-41 & 06 & 12\end{array}$ & 8,9 & $\mathrm{~A} 1 \mathrm{~V}$ & $\mathrm{CrEu}$ & $\mathrm{A} 2 \mathrm{Cr} \mathrm{Eu}$ & 9,4 \\
\hline 189963 & BD-06 5346 & 195738,7 & -064235 & 9,6 & $\mathrm{~A} 3 / 5$ II & $\mathrm{SrCrEu}$ & $\mathrm{A} 0 \mathrm{Sr} \mathrm{Cr}$ & \\
\hline 191000 & BD-04 5022 & 200230,5 & -042520 & 9,1 & B9 IV & $\mathrm{Si}$ & $\mathrm{Ao} \mathrm{Si}$ & \\
\hline 192723 & $\mathrm{CO}-28 \quad 16547$ & 201113,3 & $\begin{array}{lll}-28 & 48 & 22\end{array}$ & 9,8 & AO III/IV & $\mathrm{Si}$ & $\mathrm{AO} \mathrm{Si}$ & 10,4 \\
\hline
\end{tabular}

$\left(^{*}\right)$... without line notation by Bidelman; ? ... doubtful

Jaschek \& Jaschek (1990). It shows that $55.2 \%$ of these stars exhibit only significant Silines, $7.4 \%$ Si-Cr-Sr, $33.9 \%$ Sr-Cr-Eu and $1.4 \%$ only Sr lines. Table 1 lists the discrepant objects from all investigated catalogues.

\subsection{Distribution in the galactical plane}

The sample was further divided in a hotter $\mathrm{Si}$ and $\mathrm{Si}+$ as well as a $\mathrm{Sr}$ and $\mathrm{SrCrEu}$ group to investigate the distribution of the stars in galactic coordinates. Figure 1 shows the results for these two subgroups. First, we can conclude that the CP2 phenomenon is not clustered in specific regions. The well known effect that the hotter Si-stars are more concentrated in the galactic plane is also visible in Figure 1. For more clarity the region about the galactic plane $\left(-30^{\circ}<b<25^{\circ}, 240^{\circ}<l<360^{\circ}\right)$ was divided in rectangular fields $\left(l=10^{\circ}\right.$ and $\left.b=5^{\circ}\right)$ and the stars in these fields were counted. Figure 2 shows the number of stars vs. the marked fields.

A similar study for the Northern sky is in preparation.

\subsection{Photometric values}

The Geneva index $\Delta(V 1-G)$ and the $\Delta a$ system are capable of identifying CP2 stars photometrically. They were both developed to measure the flux depression at $\lambda 5200$. The spectral types of our sample were checked with published photometrical values of both systems. The photometric measurements were taken from the literature as referenced in Rode-Paunzen (2003)

Nearly three decades ago, Maitzen (1976) introduced the narrow-band, three filter $\Delta a$ photometric system to investigate the flux depression at $\lambda 5200$ which samples the depth of this flux depression by comparing the flux at the center $\left(\lambda 5220, g_{2}\right)$, with the adjacent regions $\left(\lambda 5000, g_{1}\right.$ and $\left.\lambda 5500, y\right)$ using a bandpass of $130 \AA$. The respective index was introduced as:

$$
a=g_{2}-\left(g_{1}+y\right) / 2
$$

Since this quantity is slightly dependent on temperature (increasing towards lower tem- 
Table 3. Summary of results for true CP2 stars in our program clusters; HD 65987 and $\mathrm{CP}-60981$ are binary systems for which we list the values for the primary component. The errors in the final digits of the corresponding quantity are given in parenthesis; $\tau$ is the age of the star, $\tau_{H R}$ the time for a star on the Main Sequence and $\tau_{C l}$ the known age of the individual cluster.

\begin{tabular}{llllccccc}
\hline Cluster & $\begin{array}{c}{[\mathrm{Fe} / \mathrm{H}]} \\
{[\mathrm{dex}]}\end{array}$ & Object & $\log T_{\text {eff }}$ & $\log L / L_{\odot}$ & $M / M_{\odot}$ & $\begin{array}{c}\tau \\
{[\mathrm{Myr}]}\end{array}$ & $\begin{array}{c}\tau_{H R} \\
{[\mathrm{Myr}]}\end{array}$ & $\tau_{C l} / \tau_{H R}$ \\
\hline NGC 2451 A & -0.26 & HD 63041 & $4.155(19)$ & $2.26(8)$ & $3.58(11)$ & 20 & 215 & 0.23 \\
NGC 2516 & -0.23 & HD 65712 & $4.002(19)$ & $1.42(12)$ & $2.16(9)$ & 90 & 870 & 0.12 \\
& & HD 65987 & $4.107(19)$ & $2.31(16)$ & {$[3.18]$} & {$[105]$} & {$[295]$} & {$[0.36]^{1}$} \\
& & HD 66295 & $4.039(19)$ & $1.64(12)$ & $2.44(10)$ & 95 & 615 & 0.17 \\
& & HD 66318 & $3.979(15)$ & $1.29(81)$ & $2.00(5)$ & 140 & 1100 & 0.10 \\
& & CP-60944A & $4.104(19)$ & $2.10(12)$ & $3.14(13)$ & 105 & 305 & 0.33 \\
& & CP-60944B & $4.096(19)$ & $1.96(12)$ & $3.03(12)$ & 105 & 340 & 0.32 \\
IC 2391 & & CP-60978 & $4.076(19)$ & $1.90(12)$ & $2.82(7)$ & 110 & 410 & 0.26 \\
IC 2602 & & CP-60981 & $3.975(15)$ & $1.51(8)$ & {$[1.95]$} & {$[100]$} & {$[1180]$} & {$[0.10]^{2}$} \\
& -0.04 & HD 74169 & $3.998(19)$ & $1.41(7)$ & $2.25(10)$ & $<10$ & 775 & 0.07 \\
& & HD 74535 & $4.418(19)$ & $2.33(7)$ & $3.80(11)$ & 35 & 185 & 0.29 \\
& & HD 92385 & $4.043(19)$ & $1.69(6)$ & $2.47(6)$ & 95 & 590 & 0.05 \\
& & HD 92664 & $4.176(19)$ & $2.47(6)$ & $3.99(12)$ & 45 & 165 & 0.18 \\
\hline
\end{tabular}

${ }^{1} \ldots$ probable binary; ${ }^{2} \ldots$ binary

Table 4. Data about our program clusters. The errors in the final digits of the corresponding quantity are given in parenthesis.

\begin{tabular}{lcccc}
\hline Name & $\begin{array}{c}\text { NGC 2451 A } \\
\text { C0743-378 }\end{array}$ & $\begin{array}{c}\text { NGC 2516 } \\
\text { C0757-607 }\end{array}$ & $\begin{array}{c}\text { IC 2391 } \\
\text { C0838-528 }\end{array}$ & $\begin{array}{c}\text { IC 2602 } \\
\text { C1041-641 }\end{array}$ \\
\hline$l / b$ & $252 /-7$ & $274 /-16$ & $270 /-7$ & $290 /-5$ \\
$E(B-V)$ & 0.01 & 0.13 & 0.01 & 0.04 \\
$\pi(\mathrm{Lit})$ & $5.31(19)$ & $2.89(21)$ & $6.85(22)$ & $6.58(16)$ \\
$\pi(\mathrm{our})$ & $5.35(21)$ & $2.69(22)$ & $6.98(18)$ & $6.99(15)$ \\
$d[\mathrm{pc}]$ & $187(7)$ & $372(33)$ & $143(3)$ & $143(3)$ \\
$\log t$ & 7.70 & 8.00 & 7.70 & 7.46 \\
{$[\mathrm{Fe} / \mathrm{H}]$} & -0.26 & -0.23 & -0.04 & -0.20 \\
$\mathrm{n}(\mathrm{CP} 2)$ & 1 & 8 & 2 & 2 \\
\hline
\end{tabular}

peratures), the intrinsic peculiarity index had to be defined as

$$
\Delta a=a-a_{0}\left[(b-y) ;(B-V) ;\left(g_{1}-y\right)\right]
$$

i.e., the difference between the individual $a$-values and the $a$-values of non-peculiar stars of the same colour. The locus of the $a_{0}$-values has been called normality line. It was shown that virtually all peculiar stars with magnetic fields (CP2 stars) have positive $\Delta a$ values up to 95 mmag. Extreme cases of the CP1 and CP3 group may exhibit marginally peculiar positive $\Delta a$ values whereas Be/Ae and $\lambda$ Bootis stars exhibit significant negative ones. Note that $\left(g_{1}-y\right)$ shows an excellent correlation with $(b-y)$ and can be used as an index for the effective temperature.

\section{On the evolutionary status of chemically peculiar stars}

The question, if the CP-phenomenon is a evolutionary effect, was discussed in the last years. We present evidence that the magnetic chemically peculiar stars (CP2) of the upper Main Sequence already occur at very early stages of the stellar evolution significantly before they reach $30 \%$ of their lifetime. A detailed description is given in Pöhnl et al. (2003). 


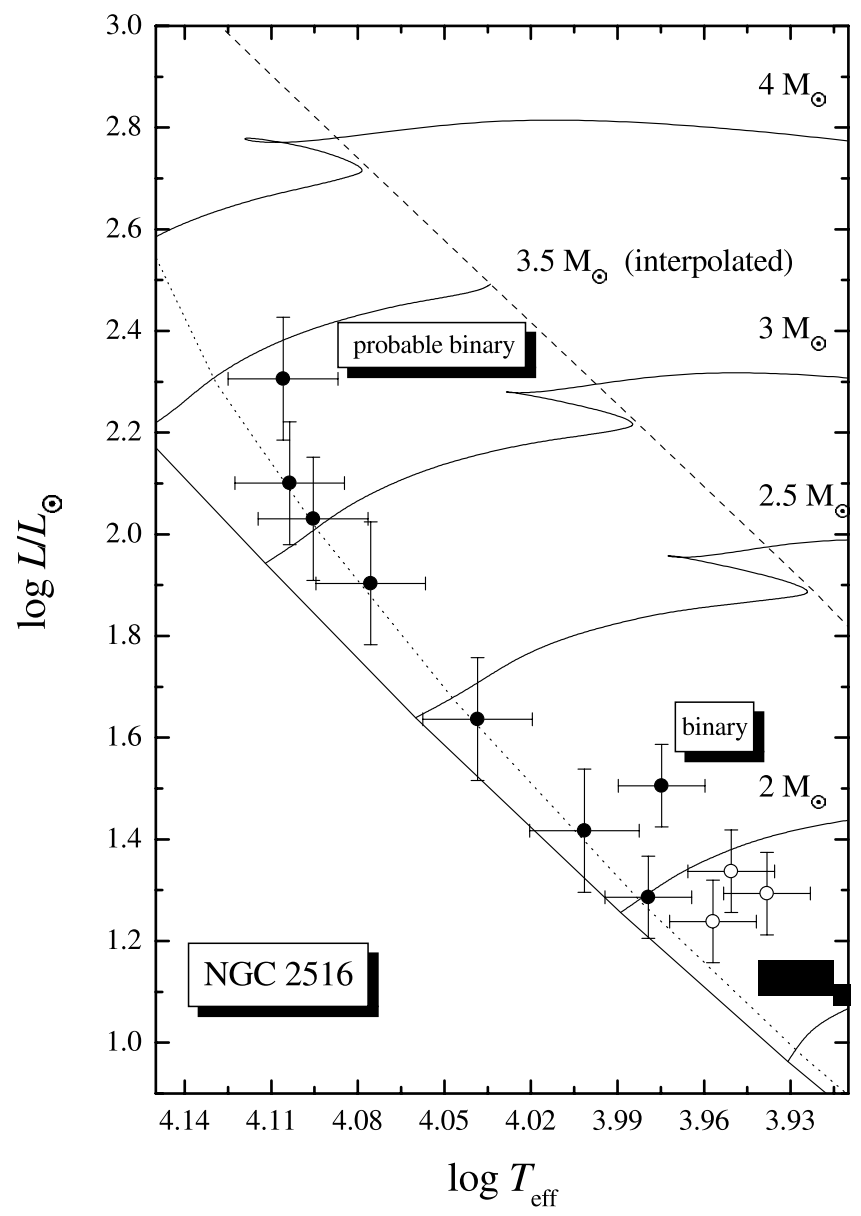

Figure 3. The location of the CP2 stars for NGC 2516. Filled circles are well established CP2 objects and open circles are doubtful cases. The dotted line is the isochrone for $\log t=8.00$ and $[\mathrm{Fe} / \mathrm{H}]=-0.23$ dex taken from Schaller et al. (1992) whereas the dashed line denoted the Terminal Age Main Sequence; the evolutionary tracks for individual masses are interpolated.

We have used the measurements and calibrations of the Geneva 7-color photometric system to derive effective temperatures and luminosities. Taking into account the overall metallicities of the individual clusters, isochrones and evolutionary tracks were used to estimate ages and masses for the individual objects. The derived ages (between 10 and $140 \mathrm{Myr}$ ) are well in line with those of the corresponding cluster and further strengthens the membership of the investigated CP2 stars. In total $13 \mathrm{CP} 2$ stars can be found in four open clusters: IC 2391, IC 2602, NGC 2451A and NGC 2516.

Figure 3 shows the location of the CP2 stars in NGC 2516 together with the isochrone for $\log t=8.00$ and $[\mathrm{Fe} / \mathrm{H}]=-0.23$ dex and the evolutionary tracks for individual masses. All but two (HD 65987 and $\mathrm{CP}-60^{\circ}$ 981) lie very well on the apparent Main Sequence of this cluster and are members of it. The two deviating objects are both binaries. Their location above the corresponding isochrone can be very well reproduced taking the binarity into account. The masses and ages for the visual binary system $\mathrm{CP}-60^{\circ} 944 \mathrm{~A}+\mathrm{B}$, both in very good agreement with the values from Debernardi \& North (2001), infer that they are physically coupled. The mean value of the age for all six single CP2 stars is 106(17) Myr which is in excellent agreement with the overall age of this cluster (100 Myr). The 
values and properties of the investigated open clusters are listed in Table 3 and $4 . \tau$ is the age of the star, $\tau_{H R}$ the time for a star on the Main Sequence and $\tau_{C l}$ the known age of the individual cluster. As a confirmation, Bagnulo et al. (2003) have discovered a strong magnetic field of $14.5 \mathrm{kG}$ in the NGC 2516 star HD 66318 . They estimate that this star has completed only about $16(5) \%$ of its main sequence life.

\section{Conclusion}

The Michigan Catalogue was studied to derive the distribution of CP2 stars in the galactic field in the Southern hemisphere. A cross check with other catalogues results that in the Michigan Catalogue, 3.6\% of all stars in the relevant spectral region are CP2 stars. Any discrepant classified stars could be verified photometrically in the $\Delta a$ system. The distribution of CP2 stars does not show any particular preferred region in the southern hemisphere.

The location of all investigated CP2 stars within the relevant Hertzsprung-Russelldiagrams qualify them as being members of the corresponding aggregate. Furthermore, their ages $(10<$ ages $<140 \mathrm{Myr})$ are within the expected error of the overall age of the individual open cluster. This proves that CP2 stars do exist at very young evolutionary stages clearly before they have reached $30 \%$ of their Main Sequence lifetime.

\section{References}

Bagnulo, S., Landstreet, J.D., Lo Curto, G., Szeifert, T., Wade, G.A. 2003, A\&A A 403, 645

Bidelman, W.P., MacConnell, D.J. 1973, AJ 78, 678

Debernardi, Y., North, P. 2001, A\&A 372, 204

Houk, N. 1978, University of Michigan Catalogue of Two-Dimensional Spectral Types for the HD stars, Volume II, Astronomy Dept., Univ. Mich., Ann Arbor, Michigan

Houk, N. 1982, University of Michigan Catalogue of Two-Dimensional Spectral Types for the HD stars, Volume III, Astronomy Dept., Univ. Mich., Ann Arbor, Michigan

Houk, N., Cowley, A.P. 1975, University of Michigan Catalogue of Two-Dimensional Spectral Types for the HD stars, Volume I, Astronomy Dept., Univ. Mich., Ann Arbor, Michigan

Houk, N., Smith-Moore, M. 1988, University of Michigan Catalogue of Two-Dimensional Spectral Types for the HD stars, Volume IV, Astronomy Dept., Univ. Mich., Ann Arbor, Michigan

Houk, N., Swift, C. 1999, University of Michigan Catalogue of Two-Dimensional Spectral Types for the HD Stars, Volume V, Astronomy Dept., Univ. Mich., Ann Arbor, Michigan

Jaschek, C., Jaschek, M. 1990, The Classification of Stars, Cambridge University Press, Cambridge

Maitzen, H.M. 1976, A\&̈A 51, 223

Pöhnl, H., Maitzen, H.M., Paunzen, E. 2003, A\&A 402, 247

Renson, P. 1991, Catalogue Général des Étoiles Ap et Am, Institut d'Astrophysique Université Liège

Rode-Paunzen, M. 2003, Statistische Studien chemisch pekuliarer Sterne der oberen Hauptreihe, Diploma thesis, University Vienna

Rufener, F. 1988, Catalogue of Stars meashured in the Geneva Observatory Photometric system (fourth edition), Observatoire Genéve

Schaller, G., Schaerer, G., Meynet, G., Maeder, A. 1992, A\&AS 96, 269 